\title{
Plasma Circular RNA Panel to Diagnose Hepatitis B Virus-Related Hepatocellular Carcinoma
}

3 Authors' Names:

4 Jian Yu, ${ }^{1,2,3,} *$ Meng-chao Wang,, 2, 3, * Wen-bing Ding, ${ }^{1,2,3, *}$ Xing-gang Guo, ${ }^{1,2,3, *}$ Jian Xu ${ }^{4, *}$ Qing-guo

5 Xu, ${ }^{1,2,3}$ Yuan Yang, ${ }^{1,2,3}$ Shu-han Sun, ${ }^{5,6}$ Jing-feng Liu ${ }^{7, \#}$, Lun-xiu Qin ${ }^{8, \#}$, Hui Liu, ${ }^{1,2,3, \#}$ Fu Yang, ${ }^{5,6 \#}$ and

6 Wei-ping Zhou ${ }^{1,2,3, \#}$

\section{Affiliations:}

8 1. The Third Department of Hepatic Surgery, Eastern Hepatobiliary Surgery Hospital, Second Military 9 Medical University, Shanghai 200438, China

10 2. Key Laboratory of Signaling Regulation and Targeting Therapy of Liver Cancer (SMMU) , Ministry of 11 Education. Shanghai 200438, China

12 3. Shanghai Key Laboratory of Hepatobiliary Tumor Biology (EHBH), Shanghai 200438, China

13 4. Department of Laboratory Diagnosis, Changhai Hospital, Second Military Medical University, Shanghai $14 \quad 200438$, China

15 5. Department of Medical Genetics, Second Military Medical University, Shanghai 200433, China

16 6. Shanghai Key Laboratory of Cell Engineering (14DZ2272300), People's Republic of China

17 7. Mengchao Hepatobiliary Hospital, Fujian Medical University, Fuzhou, 350025, China

18 8. Department of General Surgery, Huashan Hospital \& Cancer Metastasis Institute \& Institutes of 19 Biomedical Sciences, Fudan University, Shanghai 200040, China

$20 \quad{ }^{\#}$ Corresponding authors.

$21 \quad *$ These five authors contributed equally to this work.

\section{Contact information:}

23 1. Wei-Ping Zhou, M.D., Ph. D, The Third Department of Hepatic Surgery, Eastern Hepatobiliary Surgery

24 Hospital, 225 Changhai Road, 200438, Shanghai, China. E-mail: ehphwp3@126.com. Fax:

$25+86-021-81875529$

26 2. Fu Yang, Ph.D. Department of Medical Genetics, Second Military Medical University, 800 Xiangyin 
1 Road, Shanghai 200433, P. R. China. E-mail: yangfusq1997@ smmu.edu.cn.

2 3. Hui Liu, M.D., Ph. D, The Third Department of Hepatic Surgery, Eastern Hepatobiliary Surgery Hospital,

3225 Changhai Road, 200438, Shanghai, China. E-mail: liuhuigg@ hotmail.com.

4 4. Lun-xiu Qin, M.D., Ph. D, Department of General Surgery, Huashan Hospital \& Cancer Metastasis

5 Institute \& Institutes of Biomedical Sciences, Fudan University, Shanghai, China. E-mail:

6 qinlx@fudan.edu.cn.

7 5. Jing-Feng Liu, M.D., Ph. D, Mengchao Hepatobiliary Hospital, Fujian Medical University, Fuzhou,

8 350025, China. E-mail: drjingfeng@126.com.

$9 \quad$ Email address of all authors:

10 Jian Yu: $1274432278 @ q q . c o m$

11 Meng-chao Wang: diaskeyanna@live.com

12 Wen-bing Ding: dwb2017dejyd@163.com

13 Xing-gang Guo: guoxinggangdf@qq.com

14 Jian Xu: xujian20130310@163.com

15 Qing-guo Xu: xuqingguo8866@126.com

16 Yuan Yang: yy@smmu.edu.cn

17 Shu-han Sun: 18052256005@163.com

18 Jing-feng Liu: drjingfeng@126.com

19 Lun-xiu Qin: qinlx@fudan.edu.cn

20 Hui Liu: liuhuigg@ hotmail.com

21 Fu Yang: yangfusq1997@ smmu.edu.cn

22 Wei-ping Zhou: ehphwp3@126.com

23 Key words: Circular RNA, CircPanel, Diagnosis, Hepatocellular carcinoma, Plasma 


\section{Conflict of interest:}

2 The authors who have taken part in this study declared that they do not have anything to disclose regarding

3 funding or conflict of interest with respect to this manuscript.

4 Financial Support: This work was supported by the Shanghai Sailing Program (19YF1459600); National

5 Key Research and Development Program of China (2016YFC1302303); National Key Basic Research

6 Program of China (2014CB542102); Science Fund for Creative Research Groups, NSFC, China (81521091);

7 State key infection disease project of China (2018ZX10732202-002-005); National Human Genetic

8 Resources Sharing Service Platform (2005DKA21300); National Natural Science Foundation of China 9 (81372207, 81472689, 81472691, 81502375, 81672345, 81772529); State Key Program of National Natural

10 Science Foundation of China (81330037).

11 Authors' contributions:

12 Conception and design: Wei-ping Zhou, Fu Yang, Hui Liu, Lun-xiu Qin, and Jing-feng Liu

13 Financial support: Wei-ping Zhou, Fu Yang, Hui Liu and Shu-han Sun

14 Administrative support: Wei-ping Zhou, Fu Yang and Lun-xiu Qin

15 Provision of study materials or patients: Yuan Yang, Jian Xu and Jing-feng Liu

16 Collection and assembly of data: Jian Yu, Meng-chao Wang, Wen-bing Ding, Xing-gang Guo, Jian Xu and

17 Qing-guo Xu

18 Data analysis and interpretation: Jian Yu, Meng-chao Wang, Wen-bing Ding, Xing-gang Guo, Jian Xu and

19 Lun-xiu Qin

20 Manuscript writing: All authors

21 Final approval of manuscript: All authors

Abbreviations: AFP, alpha-fetoprotein; ALT, alanine aminotransferase; ANL, adjacent noncancerous liver;

23 AUC, area under the receiver operating characteristic curve; BCLC, Barcelona Clinic Liver Cancer; CHB, 
1 chronic hepatitis B; CI, confidence interval; CircPanel, circRNA panel containing three circRNAs

2 (hsa_circ_0000976, hsa_circ_0007750 and hsa_circ_0139897); circRNA, circular RNA; HBsAg, hepatitis B

3 surface antigen; HBV, hepatitis B virus; HCC, hepatocellular carcinoma; PCR, polymerase chain reaction;

4 qRT-PCR, quantitative reverse transcription polymerase chain reaction; ROC, receiver operating

5 characteristics.

6 
Abstract

2 To explore whether plasma circular RNAs (circRNAs) can diagnose hepatitis B virus (HBV)-related

3 hepatocellular carcinoma (HCC), microarray and qPCR were used to identify plasma circRNAs that were

4 increased in HCC patients compared with controls (including healthy controls, chronic hepatitis B,

$5 \quad$ HBV-related liver cirrhosis and HCC patients). A logistic regression model was constructed using a training

6 set $(n=313)$ and then validated using another two independent sets ( $n=306$ and 526, respectively). Area

7 under the receiver operating characteristic curve (AUC) was used to evaluate diagnostic accuracy. We

8 identified a plasma circRNA panel (CircPanel) containing three circRNAs (hsa_circ_0000976,

9 hsa_circ_0007750 and hsa_circ_0139897) that could detect HCC. CircPanel showed a higher accuracy than

10 AFP (alpha-fetoprotein) to distinguish individuals with HCC from controls in all three sets (AUC 0.863 [95\%

11 CI $0.819-0.907]$ vs 0.790 [0.738-0.842], $\mathrm{P}=0.036$ in training set; 0.843 [0.796-0.890] vs 0.747 [0.691-

$120.804], \mathrm{P}=0.011$ in validation set 1 and $0.864[0.830-0.898]$ vs 0.769 [0.728-0.810], $\mathrm{P}<0.001$ in validation

13 set 2). CircPanel also performed well in detecting Small-HCC (solitary, $\leq 3 \mathrm{~cm}$ ), AFP-negative HCC and

14 AFP-negative Small-HCC.

15 


\section{Significance of this study}

\section{What is already known about this subject?}

3 1. The diagnostic accuracy of alpha-fetoprotein (AFP) in detecting hepatocellular carcinoma (HCC) is

4 unsatisfactory.

5 2. Circular RNA (circRNA) expression profiles in HCC and adjacent nontumor liver tissues are significantly

6 different.

7 3. Plasma circRNAs are enriched, stable and can be biomarkers for various diseases.

\section{What are the new findings?}

9 1. The expression of circRNAs in the plasma from HCC patients and chronic hepatitis B is significantly 10 different.

11 2. Plasma circRNA panel (CircPanel, including hsa_circ_0000976, hsa_circ_0007750 and hsa_circ_0139897) 12 has a higher accuracy than AFP to distinguish individuals with HCC or Small-HCC (solitary, $\leq 3 \mathrm{~cm}$ ) from 13 controls (healthy controls, chronic hepatitis B and HBV-related liver cirrhosis).

14 3. CircPanel also performs well in diagnosing AFP-negative HCC and AFP-negative Small-HCC.

15 How might it impact on clinical practice in the foreseeable future?

16 Plasma CircPanel can be a diagnostic biomarker in detecting HCC and improves the diagnostic accuracy. 
bioRxiv preprint doi: https://doi.org/10.1101/576751· this version posted March 14,2019 . The copyright holder for this preprint (which was not certified by peer review) is the author/funder, who has granted bioRxiv a license to display the preprint in perpetuity. It is made available under aCC-BY-NC-ND 4.0 International license.

\section{Introduction}

2 Hepatocellular carcinoma (HCC), largely attributable to chronic hepatitis B virus (HBV) infection, is

3 the second most common gastrointestinal solid tumors and remains the second leading cause of

4 cancer-related death in China ${ }^{1}$. The high mortality of $\mathrm{HCC}$ is due partly to the fact that early-stage HCC

5 shows no obvious symptoms and the diagnostic accuracy of AFP (alpha-fetoprotein, a serum biomarker for

6 the diagnosis of HCC in clinical use) is unsatisfactory. The sensitivity and specificity of high serum AFP for

7 HCC were reported to range from $39-64 \%$ and $76-91 \%$, respectively. ${ }^{2-4}$ Therefore, a novel biomarker for

8 the detection of HCC, especially early-stage HCC, need to be identified.

9 Circular RNAs (termed circRNAs) are covalently closed, single-stranded and stable transcripts. ${ }^{5}$ In our 10 previous study, we demonstrated that circRNA expression profiles in HCC and adjacent nontumor liver 11 tissues are significantly different and circular RNA cSMARCA5 inhibits the growth and metastasis of 12 HCC. $^{6}$ Furthermore, it has been reported that plasma circRNAs are enriched, stable and can be biomarkers 13 for non-small cell lung cancer and systemic lupus erythematosus ${ }^{7}$. In this study, using microarray and 14 qRT-PCR (quantitative real-time polymerase chain reaction), we tried to explore whether plasma circRNAs 15 can be biomarkers to diagnose HBV-related HCC (referred to below as HCC). 


\section{Patients and Methods}

\section{Study Design and Participants}

The study design is listed in Figure 1. In total, 1195 plasma samples, 40 paired HCC and adjacent

4 noncancerous liver (ANL) tissues were collected from three hospitals in China. The recruited participants

5 were defined as healthy individuals, patients with chronic hepatitis B (CHB), patients with HBV-related

6 liver cirrhosis (referred to below as liver cirrhosis), or patients with HCC by medical doctors, according to

7 eligibility criteria listed in Supplementary Table 1.

8 The plasma, HCC and ANL tissues in the discovery and training sets were collected between July 2016

9 to June 2017 at the Shanghai Eastern Hepatobiliary Surgery Hospital, Second Military Medical University,

10 Shanghai, China. The plasma in validation set 1 was collected between September 2016 to July 2017 at the

11 Shanghai Changhai Hospital, Second Military Medical University, Shanghai, China. The plasma in

12 validation set 2 was collected between February 2016 to May 2018 at the Mengchao Hepatobiliary Surgery

13 Hospital, Fujian Medical University, Fuzhou, China. The details of the clinicopathological characteristics of

14 the participants are listed in Supplementary Table 2. In addition, for the 40 HCC patients undergoing

15 hepatectomy in the training set, we also collected their plasma at the 30th day after hepatectomy, their HCC and paired ANL tissues. The details of the clinicopathological characteristics of these $40 \mathrm{HCC}$ patients are

17 listed in Supplementary Table 3.

Human specimen collection was approved by the ethics committee of each hospital. Written informed

consent was obtained from each patient according to the policies of the committee.

\section{RNA Isolation}

For the HCC cell lines and the HCC and ANL tissues, the total RNA was extracted using RNAiso Plus (Takara, Code No. 9109) according to the manufacturer's instructions.

For the plasma, the total RNA was extracted using the TRIzol ${ }^{\text {TM }}$ LS Reagent (ThermoFisher, Code No. 
1 10296010) according to the manufacturer's instructions. During the isopropanol precipitation, glycogen

2 (catalogue number AM9510, Ambion/Applied Biosystems, Foster City, CA) was added as a coprecipitant

3 (final concentration of $100 \mu \mathrm{g} / \mathrm{mL}$ ) to enhance the RNA precipitation.

4 CircRNA Microarray Expression Profiling

The total RNAs extracted from the plasma of five HCC patients and five CHB patients were used for

6 microarray analysis as described previously. ${ }^{8,9}$ In brief, the RNAs were digested, amplificated, labelled and

7 hybridized onto the microarray (CapitalBio Technology Human CircRNA Array, Version 2.0). Differential

8 expression analysis of circRNAs was performed using GeneSpring software V13.0 (Agilent). We used

9 threshold values of $\geq 2$ or $\leq-2$-fold change and a t-test $\mathrm{P}$-value $<0.05$. The differentially expressed

10 circRNAs are listed in Supplementary Table 4. The data were Log2 transformed and median centered by

11 genes using the Adjust Data function of CLUSTER 3.0 software and were then further analyzed by

hierarchical clustering with average linkage. ${ }^{10}$ Finally, we performed tree visualization by using Java

13 Treeview (Stanford University School of Medicine, Stanford, CA, USA).

\section{Reverse Transcription}

Total RNA from both tissues or plasma was reversely transcribed using M-MLV Reverse Transcriptase Kit (ThermoFisher, Code No. 28025021) according to the manufacturer's instructions.

\section{Quantitative Reverse-transcriptase Polymerase Chain Reaction (qRT-PCR)}

The qRT-PCR, using SYBR ${ }^{\circledR}$ Premix Ex Taq ${ }^{\text {TM }}$ II (Tli RNaseH Plus) and ROX plus (Takara, Code No. RR82LR), was performed on the StepOneTM Real-Time PCR System (Applied Biosystems, Foster City,

CA). The PCR primers for $\beta$-actin and the four candidate circRNAs (hsa_circ_0000976, hsa_circ_0003506, hsa_circ_0007750 and hsa_circ_0139897) are listed in Supplementary Table 5. The primers for the circRNAs were divergent and circRNA specific. (Supplementary Figure 1A)

For the HCC and ANL tissues, ACTB was employed as the endogenous control, and the relative 
1 expression was calculated using the comparative $\Delta \Delta \mathrm{Ct}$ method.

Since there is no accepted endogenous control for the quantitation of mRNAs/circRNAs in plasma, we

4 previously described. ${ }^{11-13}$ Briefly, the PCR products were amplified from human pooled plasma cDNA using

used absolute quantitation when detecting the expression of the candidate circRNAs in the plasma as

the primers of the four candidate circRNAs, respectively. Subsequently, the four PCR products were cloned into pUC57 vector, respectively. The resulting constructs were verified by direct sequencing and serially

diluted from $5 \times 10^{7}$ copies/ $\mu$ l to 5 copies $/ \mu 1$, respectively. Those diluted constructs were run in parallel with the samples under identical qPCR conditions and amplified with the same set of primers. A standard curve was generated by plotting the cycle threshold as a function of $\log _{10}$ concentration of the serial diluted controls (Supplementary Figure 1B-E). The relative amount of cDNA of a particular template was extrapolated from the standard curve using the LightCycler software 3.0 (Bio-Rad).

\section{Statistical Analysis}

All statistical analyses were performed using SPSS version 23.0 software (SPSS, Inc., Chicago, IL).

For comparisons, chi-squared test, Student's t test, Wilcoxon signed-rank test, Mann-Whitney U test and Kruskal-Wallis H test were performed, as appropriate. Correlations were measured by Spearman correlation analysis. The optimal cut-off values of the expression of the candidate circRNAs in plasma were determined by a ROC curve (Euclidean distance) analysis in Cutoff Finder ${ }^{14}$ (http://molpath.charite.de/cutoff/). Binary logistic regression was used to build the diagnostic model CircPanel (circRNA panel, including hsa_circ_0000976, hsa_circ_0007750 and hsa_circ_0139897) as described previously. ${ }^{12,15,16}$ Area under the receiver operating characteristic curve (AUC) was used to evaluate diagnostic accuracy. The comparation of AUC was performed by the pROC package of R software (version 3.0.1). ${ }^{15,17}$ All P values were two sided. It was considered to be statistically significant when $\mathrm{P}<0.05$.

The remaining methods are described in the Supplementary Data 1. 
bioRxiv preprint doi: https://doi.org/10.1101/576751; this version posted March 14, 2019. The copyright holder for this preprint (which was not certified by peer review) is the author/funder, who has granted bioRxiv a license to display the preprint in perpetuity. It is made available under aCC-BY-NC-ND 4.0 International license. 


\section{Results}

\section{Identification of Circular RNAs By Microarray and PCR in Human Plasma Samples}

$4 \quad$ HCC patients and five CHB patients (discovery set). Among the 371 differentially expressed circRNAs, 326

5 were upregulated and 45 were downregulated in the plasma from HCC patients compared with that from

6 CHB patients (Supplementary Table 4, Supplementary Figure 2A). We hypothesized that circRNAs in HCC

$7 \quad$ tissues could be secreted into plasma. By overlapping the 326 upregulated plasma circRNAs and the 6584

8 circRNAs detected in HCC tissues from our group's previous study ${ }^{6}$, we obtained 10 candidate circRNAs

9 (Supplementary Table 4). Subsequently, we successfully validated four of these (hsa_circ_0000976,

10 hsa_circ_0003506, hsa_circ_0007750 and hsa_circ_0139897) in human plasma (Supplementary Figure

11 2B-D), human HCC tissues (Supplementary Figure 3A-C), and human HCC cell lines (HepG2 and Huh7)

12 (Supplementary Figure 4) and by qRT-PCR using circRNA-specific divergent primers, agarose gel

13 electrophoresis and Sanger sequencing. Furthermore, we found that incubating plasma at room temperature

14 for up to $24 \mathrm{~h}$ had minimal effect on the expression of these four circRNAs (Supplementary Figure 2E),

15 indicating that they were stable in plasma and could be used as a biomarker. In addition, after the treatment

16 with RNase R (a highly processive 3'-to-5' exoribonuclease that digests linear RNAs ${ }^{18}$ ), none showed

17 significant changes (Supplementary Figure 3D), which demonstrated that they were truly circular and not

18 linear.

Subsequently, HepG2 and Huh7 cells were subcutaneously implanted into the bilateral armpits of

BALB/c nude mice. Twenty-eight days later (once tumors were well established), all mice were sacrificed, and their plasma was collected for the detection of the four candidate circRNAs. As expected, agarose gel

electrophoresis following RT-PCR (Supplementary Figure 5) and Sanger sequencing (Supplementary Figure

6) showed that these four circRNAs existed in the plasma from the mice in the HepG2 and Huh7 groups but 
1 not in the control group. This proved that HCC cells secrete circRNAs into plasma.

\section{The Building of the Diagnostic Model CircPanel Based on the Training Set}

By qRT-PCR, we detected the expression of hsa_circ_0000976, hsa_circ_0003506, hsa_circ_0007750

4

and hsa_circ_0139897 in the plasma from 158 HCC patients, 53 healthy controls, 52 CHB patients and 50

HBV-induced liver cirrhosis patients (Figure 1) and found that the expression of hsa_circ_0000976,

hsa_circ_0007750 and hsa_circ_0139897 (but not hsa_circ_0003506) in the plasma from the HCC patients

was higher than that in the plasma from the healthy controls, CHB patients and liver cirrhosis patients

(Supplementary Figure 7A). For the 40 HCC patients undergoing hepatectomy in the training set, we also

detected the expression of these four circRNAs in their plasma at the 30th day after hepatectomy and in their HCC and paired ANL tissues. We found that the expression of hsa_circ_0000976, hsa_circ_0007750 and hsa_circ_0139897 (but not hsa_circ_0003506) in the plasma from the HCC patients was positively correlated with the expression in their HCC tissues (Supplementary Figure 7B) and was significantly downregulated after hepatectomy (Supplementary Figure 7C). Furthermore, the expression of hsa_circ_0000976 and hsa_circ_0007750 was higher in the HCC tissues than in the ANL tissues (Supplementary Figure 7D). Therefore, we chose hsa_circ_0000976, hsa_circ_0007750 and hsa_circ_0139897 as the candidate circRNAs for the diagnosis of HCC.

Using Cutoff Finder ${ }^{14}$ (http://molpath.charite.de/cutoff/), we determined that the best cutoff values of plasma hsa_circ_0000976, hsa_circ_0007750 and hsa_circ_0139897 for distinguishing HCC and Non-HCC (healthy controls, CHB patients and liver cirrhosis patients as a whole) were 1067, 4324 and 1108 copies/ml of plasma, respectively. Their diagnostic performance is shown in Supplementary Table 6. To improve diagnostic accuracy, using binary logistic regression, we built the diagnostic model CircPanel. The predicted probability of being detected as $\mathrm{HCC}$ by the CircPanel was calculated by: Logit $(\mathrm{P}=\mathrm{HCC})=$ $-3.502+1.920 *$ hsa_circ_0000976+2.800*hsa_circ_0007750+3.154*hsa_circ_0139897. In this equation, the 
circRNA symbol was substituted with the discretized value one when the level of the circRNA was higher

than the corresponding best cutoff value; otherwise, it was substituted with the discretized value of zero. If

the result of logit $[\mathrm{p}=\mathrm{HCC}]$ was higher than 0.5 , then the detected sample was predicted as HCC; otherwise it

4 was Non-HCC. As expected, the diagnostic accuracy of the CircPanel was higher than those of

5 hsa_circ_0000976 (AUC 0.863 [0.819-0.907] vs 0.702 [0.644-0.761], P<0.001), hsa_circ_0007750 (AUC

60.863 [0.819-0.907] vs 0.776 [0.723-0.830], $\mathrm{P}=0.012$ ) and hsa_circ_0139897 (AUC 0.863 [0.819-0.907] vs

$7 \quad 0.749[0.693-0.804], \mathrm{P}=0.001)($ Supplementary Table 6).

8

9

We also analyzed the diagnostic performance of AFP, whose recommended clinical cutoff is $20 \mathrm{ng} / \mathrm{ml}$,

in detecting HCC in the training set (Table 1). Furthermore, using the aforementioned method, we combined the CircPanel and AFP (CircPanel+AFP) to diagnosis HCC. The predicted probability of being detected as HCC by CircPanel+AFP was calculated as Logit $(\mathrm{P}=\mathrm{HCC})=-2.152+3.321 *$ CircPanel+2.241*AFP. The diagnostic performance of CircPanel+AFP was then analyzed (Table 1).

\section{The Performance of CircPanel, AFP and Their Combination for the Diagnosis of HCC}

We then detected the expression of hsa_circ_0000976, hsa_circ_0007750 and hsa_circ_0139897 in the plasma from validation set 1 (152 HCC patients, 50 healthy controls, $54 \mathrm{CHB}$ patients and $50 \mathrm{HBV}$-induced liver cirrhosis patients) and validation set 2 (290 HCC patients, 76 healthy controls, $80 \mathrm{CHB}$ patients and 80 HBV-induced liver cirrhosis patients) and analyzed the performance of the CircPanel, AFP and their combination (CircPanel+AFP) for the diagnosis of HCC.

As a result, we found that both the CircPanel and CircPanel+AFP showed a higher accuracy than AFP in distinguishing individuals with HCC from Non-HCC in all three sets (CircPanel versus AFP: AUC 0.863 [95\% CI 0.819-0.907] vs 0.790 [0.738-0.842], $\mathrm{P}=0.036$ in the training set; 0.843 [0.796-0.890] vs 0.747 [0.691-0.804], $\mathrm{P}=0.011$ in validation set 1 and $0.864[0.830-0.898]$ vs $0.769[0.728-0.810], \mathrm{P}<0.001$ in validation set 2. CircPanel+AFP versus AFP: 0.878 [0.836-0.920] vs 0.790 [0.738-0.842], $\mathrm{P}=0.010$ in the 
training set; 0.863 [0.819-0.908] vs 0.747 [0.691-0.804], $\mathrm{P}=0.002$ in validation set 1 and 0.874 [0.840-

0.907] vs 0.769 [0.728-0.810], $\mathrm{P}<0.001$ in validation set 2). In addition, the CircPanel and CircPanel+AFP

were not significantly different in distinguishing HCC from Non-HCC (Table 1, Figure 2).

Subsequently, we divided the Non-HCC group into healthy, CHB and liver cirrhosis groups and analyzed the diagnostic performance of CircPanel, AFP and CircPanel+AFP in HCC versus Healthy, HCC versus CHB and HCC versus Cirrhosis. The results were similar, especially for HCC versus CHB and HCC versus Cirrhosis (Table 1, Figure 2).

\section{The Performance of CircPanel, AFP and Their Combination for the Diagnosis of Small-HCC}

We then analyzed the performance of CircPanel, AFP and their combination (CircPanel+AFP) in the diagnosis of Small-HCC (solitary, diameter $\leq 3 \mathrm{~cm}$ ) and found that both the CircPanel and CircPanel+AFP showed a higher accuracy than AFP in distinguishing individuals with Small-HCC from Non-HCC in all three sets (CircPanel versus AFP: 0.862 [0.796-0.928] vs 0.680 [0.589-0.770], $\mathrm{P}=0.001$ in the training set; 0.838 [0.776-0.900] vs 0.699 [0.613-0.785], $\mathrm{P}=0.011$ in validation set 1 and 0.851 [0.799-0.903] vs 0.738 [0.671-0.805], $\mathrm{P}=0.009$ in validation set 2. CircPanel+AFP versus AFP: 0.873 [0.817-0.929] vs 0.680 [0.589-0.770], $\mathrm{P}=0.001$ in the training set; $0.874[0.823-0.925]$ vs $0.699[0.613-0.785], \mathrm{P}=0.001$ in validation set 1 and 0.864 [0.818-0.910] vs 0.738 [0.671-0.805], $\mathrm{P}=0.002$ in validation set 2$)$. In addition, the CircPanel and CircPanel+AFP did not show a significant difference in distinguishing Small-HCC from Non-HCC (Table 2, Figure 3).

Subsequently, we divided the Non-HCC group into healthy, CHB and liver cirrhosis groups and analyzed the diagnostic performance of the CircPanel, AFP and CircPanel+AFP in Small-HCC versus Healthy, Small-HCC versus CHB and Small-HCC versus Cirrhosis. Similar results were obtained, especially in Small-HCC versus CHB and Small-HCC versus Cirrhosis (Table 2, Figure 3). 
1 Furthermore, we analyzed the performance of the CircPanel in the diagnosis of AFP-negative HCC and

2 AFP-negative Small-HCC. The results showed that the CircPanel also had a high diagnostic accuracy (all

3 AUCs were higher than 0.800, Table 3).

4

5

6 


\section{Discussion}

It is reported that plasma circRNA hsa_circ_0001445 is a fairly accurate marker for distinguishing

3 HCC cases from healthy controls as well as liver cirrhosis or CHB patients. ${ }^{19}$ However, this was a

$4 \quad$ single-center study with limited participants (104 HCC patients, 57 cirrhosis patients, 44 CHB patients, and $5 \quad 52$ healthy controls). ${ }^{19}$

Our study is unique for the following reasons. First, to our knowledge, this is the first report to compare

7 the expression of circRNAs in the plasma from HCC and CHB patients by microarray. Furthermore, it was a

8 multicenter study with 1155 participants. Importantly, the three circRNAs in the CircPanel proved to be

9 secreted by HCC cells, and their expression in plasma was positively correlated with that in HCC tissues,

10 though the correlation coefficient was relatively low. In addition, the CircPanel showed higher accuracy than

11 AFP in distinguishing individuals with HCC or Small-HCC from the controls and performed well in 12 diagnosing AFP-negative HCC and AFP-negative Small-HCC. All of these findings make the CircPanel a 13 compelling diagnostic biomarker.

There are a few limitations in the present study. First, all of the HCC patients in this study were HBV-related. Further studies are needed to evaluate the performance of the CircPanel in diagnosing HCC caused by other factors. Second, although the expression of hsa_circ_0139897 in HCC and ANL tissues did

17 not show a significant difference, its expression in the plasma from HCC patients was positively correlated 18 with that in their HCC tissues and was significantly downregulated after hepatectomy. The reason for this 19 discrepancy is not clear at the present time and needs further exploration. One possible explanation is that 20 HCC tissues may secrete more hsa_circ_0139897 than ANL tissues. Third, a nested case-control study

21 should be performed to evaluate the diagnostic performance of the plasma CircPanel in detecting preclinical HCC. Furthermore, the follow up of the HCC patients should be continued in order to analyze the 23 relationship between the plasma CircPanel and the prognosis of HCC patients. In addition, since the 
1 expression of hsa_circ_0000976 and hsa_circ_0007750 in the HCC tissues was higher than in the ANL

2 tissues, their role in HCC progression awaits further investigation.

3 In summary, by a microarray screening and qRT-PCR in a multicenter study, we identified a plasma

4 circRNA panel (CircPanel) containing three circRNAs (hsa_circ_0000976, hsa_circ_0007750 and

5 hsa_circ_0139897) that detected HCC. The CircPanel performed better than AFP in diagnosing HCC and

6 Small-HCC and also identified AFP-negative HCC and AFP-negative Small-HCC effectively. Therefore, we

7 believe that the CircPanel can be a potential biomarker in the clinical diagnosis of HCC.

8

9 


\section{REFERENCES}

1. Torre LA, Bray F, Siegel RL, et al: Global cancer statistics, 2012. CA Cancer J Clin 65:87-108, 2015

2. Okuda H, Nakanishi T, Takatsu K, et al: Serum levels of des-gamma-carboxy prothrombin measured using the revised enzyme immunoassay kit with increased sensitivity in relation to clinicopathologic features of solitary

5 hepatocellular carcinoma. Cancer 88:544-9, 2000

3. Marrero JA, Lok AS: Newer markers for hepatocellular carcinoma. Gastroenterology 127:S113-9, 2004

4. Oka H, Tamori A, Kuroki T, et al: Prospective study of alpha-fetoprotein in cirrhotic patients monitored for development of hepatocellular carcinoma. Hepatology 19:61-6, 1994

5. Chen LL: The biogenesis and emerging roles of circular RNAs. Nat Rev Mol Cell Biol 17:205-11, 2016

6. Yu J, Xu QG, Wang ZG, et al: Circular RNA cSMARCA5 inhibits growth and metastasis in hepatocellular

carcinoma. J Hepatol 68:1214-1227, 2018

7. Li H, Li K, Lai W, et al: Comprehensive circular RNA profiles in plasma reveals that circular RNAs can be used as novel biomarkers for systemic lupus erythematosus. Clin Chim Acta 480:17-25, 2018

pre-diabetes and type 2 diabetes mellitus. Acta Diabetol 54:237-245, 2017

9. Zhao Z, Li X, Gao C, et al: Peripheral blood circular RNA hsa_circ_0124644 can be used as a diagnostic 17 biomarker of coronary artery disease. Sci Rep 7:39918, 2017 adenoma patients. Clin Cancer Res 10:1613-7, 2004

12. Li Y, Elashoff $D$, Oh M, et al: Serum circulating human mRNA profiling and its utility for oral cancer detection.

13. Wong BC, Chan KC, Chan AT, et al: Reduced plasma RNA integrity in nasopharyngeal carcinoma patients. 
$1 \quad$ Clin Cancer Res 12:2512-6, 2006

14. Budczies J, Klauschen F, Sinn BV, et al: Cutoff Finder: a comprehensive and straightforward Web application

3 enabling rapid biomarker cutoff optimization. PLoS One 7:e51862, 2012

15. Lin XJ, Chong Y, Guo ZW, et al: A serum microRNA classifier for early detection of hepatocellular carcinoma:

5 a multicentre, retrospective, longitudinal biomarker identification study with a nested case-control study. Lancet Oncol

$6 \quad 16: 804-15,2015$

16. Hua R, Tan $Y, L i Z$, et al: A Large-scale, multicenter serum metabolite biomarker identification study for the

8 early detection of hepatocellular carcinoma. Hepatology, 2017

17. Robin X, Turck N, Hainard A, et al: pROC: an open-source package for R and S+ to analyze and compare

10 ROC curves. BMC Bioinformatics 12:77, 2011

18. Jeck WR, Sharpless NE: Detecting and characterizing circular RNAs. Nat Biotechnol 32:453-61, 2014

19. Zhang X, Zhou H, Jing W, et al: The Circular RNA hsa_circ_0001445 Regulates the Proliferation and 13 Migration of Hepatocellular Carcinoma and May Serve as a Diagnostic Biomarker. Dis Markers 2018:3073467, 2018 
Discovery set

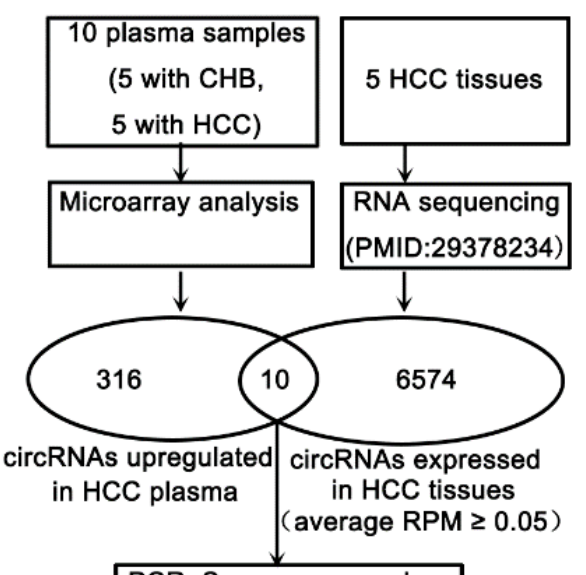

PCR, Sanger sequencing
Training set

353 plasma samples
( 53 healthy controls
52 with $\mathrm{CHB}$
50 with liver cirrhosis
158 with HCC
40 with HCC after hepatectomy)
40 paired $\mathrm{HCC}$ and ANL tissues

Validation set 1

\begin{tabular}{|l|}
\hline 06 plasma samples \\
(50 healthy controls \\
54 with $\mathrm{CHB}$ \\
50 with liver cirrhosis \\
152 with $\mathrm{HCC}$ )
\end{tabular}

Validation set 2

526 plasma samples (76 healthy controls 80 with $\mathrm{CHB}$

80 with liver cirrhosis 290 with HCC)

5 Figure 1. Study design. Abbreviations: ANL, adjacent noncancerous liver; CHB, chronic hepatitis B; HCC,

6 hepatocellular carcinoma; PCR, polymerase chain reaction; qRT-PCR, quantitative reverse-transcriptase 7 polymerase chain reaction; ROC, receiver operating characteristics. 

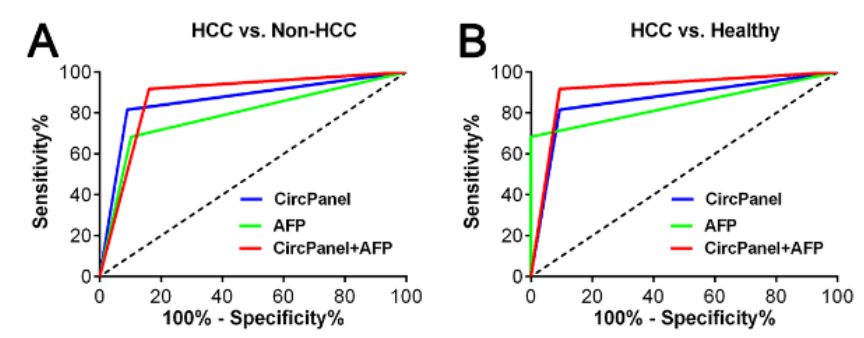

\section{Training Set}
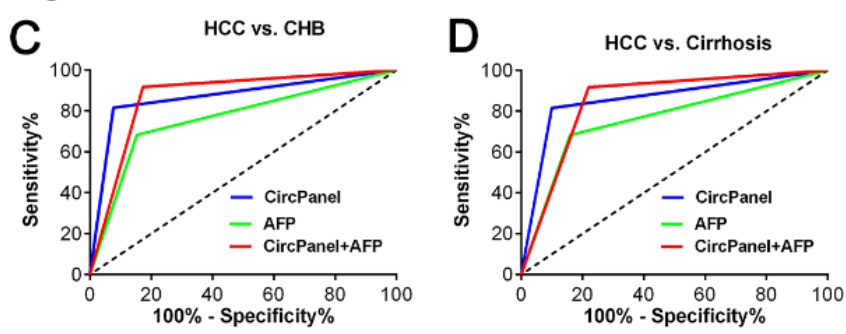

\section{Validation Set 1}
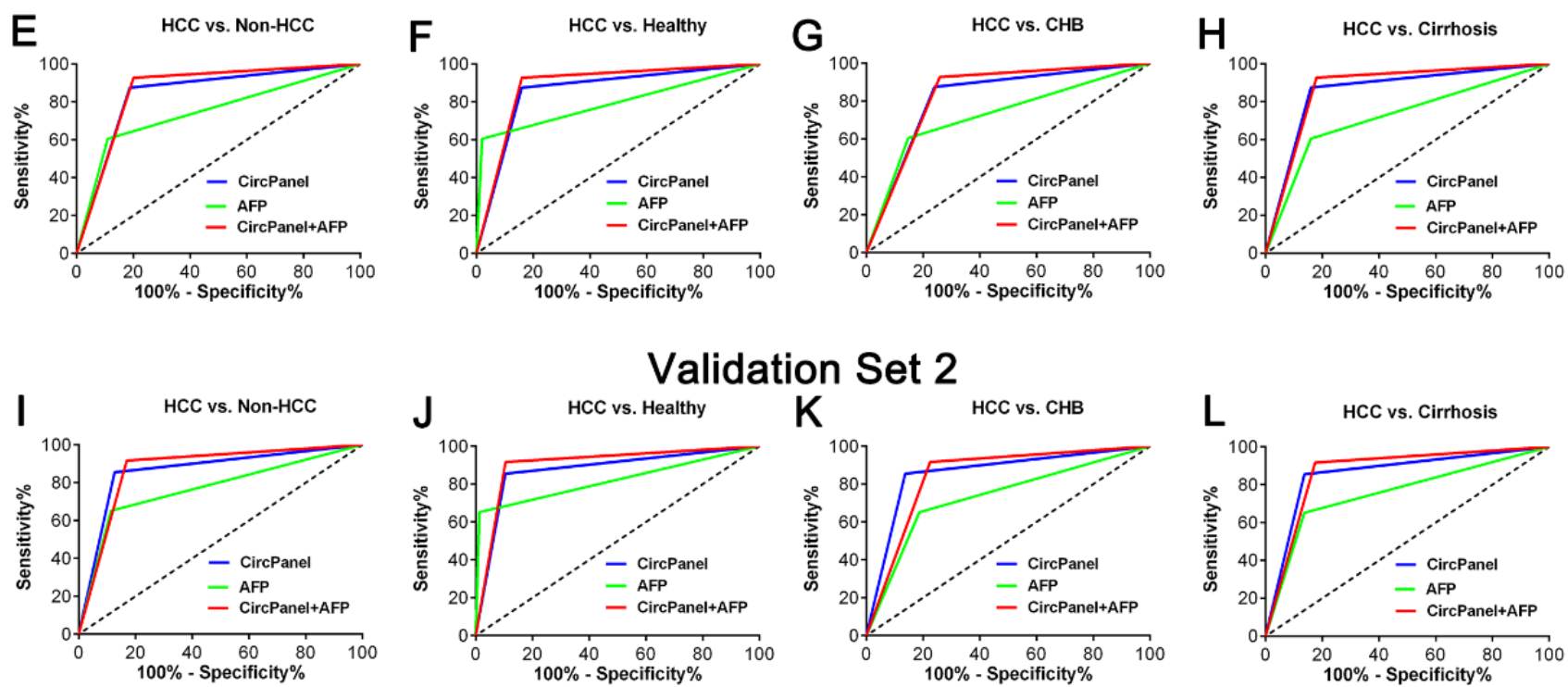

Figure 2. The performance of the CircPanel, AFP and their combination for the diagnosis of HCC in the training set (A-D), validation set 1 (E-H) and validation set 2 (I-L). The detailed diagnostic performances are listed in Table 1. Abbreviations: AFP, alpha fetoprotein; CHB, chronic hepatitis B;

7 CircPanel, circRNA panel containing three circRNAs (hsa_circ_0000976, hsa_circ_0007750 and 8 hsa_circ_0139897); HCC, hepatocellular carcinoma. 


\section{Training Set}
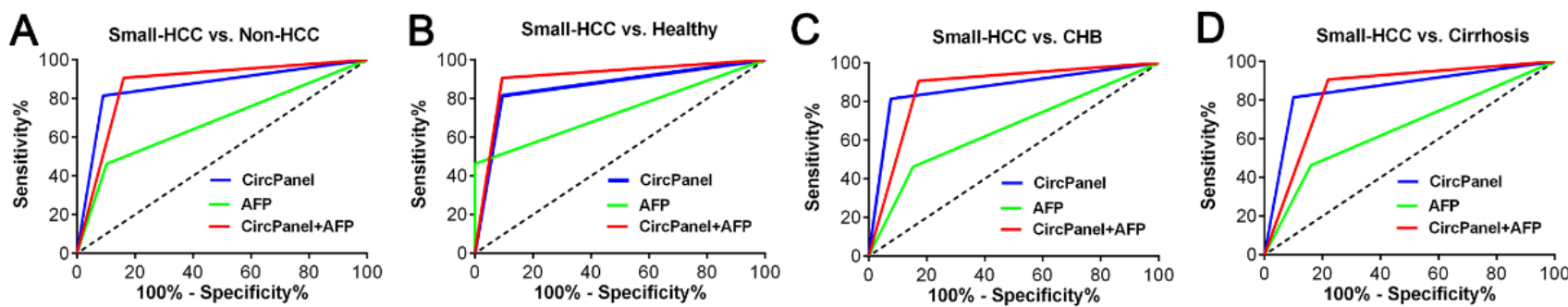

\section{Validation Set 1}
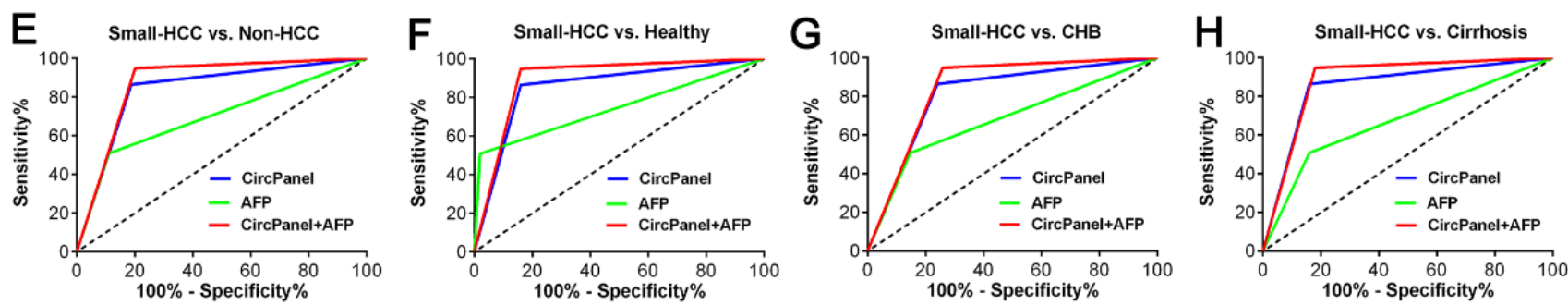

\section{Validation Set 2}
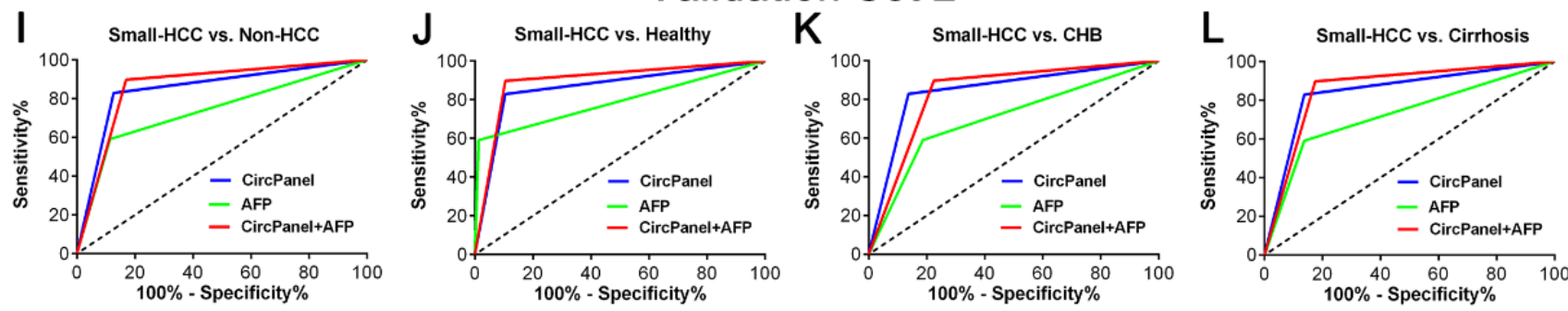

Figure 3. The performance of the CircPanel, AFP and their combination for the diagnosis of Small-HCC in the training set (A-D), validation set 1 (E-H) and validation set 2 (I-L). Thee detailed

B; CircPanel, circRNA panel containing three circRNAs (hsa_circ_0000976, hsa_circ_0007750 and hsa_circ_0139897); HCC, hepatocellular carcinoma; Small-HCC, solitary, diameter $\leq 3 \mathrm{~cm}$. 